\title{
A expansão da epidemia da leishmaniose visceral no Estado de Mato Grosso, 1998-2005
}

\author{
The spread of the visceral leishmaniasis epidemic \\ in the State of Mato Grosso, 1998-2005
}

\author{
Gustavo Leandro da Cruz Mestre ${ }^{1,2}$ e Cor Jésus Fernandes Fontes ${ }^{1,2}$
}

\begin{abstract}
RESUMO
Uma epidemia de leishmaniose visceral teve início em 1998 na Região Metropolitana de Cuiabá, capital de Mato Grosso, atingindo boje 34 (24,1\%) dos 141 municípios do estado. Entre janeiro de 1998 e dezembro de 2005, foram notificados 138 casos autóctones, predominando o sexo masculino (58\%), crianças $(51,5 \%)$ de $0-9$ anos e residentes $(66,7 \%)$ de áreas urbanas. A leishmaniose visceral canina foi identificada em 41 municípios, com soropositividade de $9 \%$ em 40.000 cães examinados. Lutzomyia longipalpis e/ou Lutzomyia cruzi foram capturadas em 14 dos 18 municípios que registraram simultaneamente leishmaniose visceral humana e canina. Os resultados indicam que a transmissão da leishmaniose visceral dissemina-se para o interior do estado, acompanhando o fluxo migratório e o processo de ocupação urbana desordenada das cidades. A presença isolada de Lutzomyia cruzi em municípios com alta incidência de casos humanos e caninos de leishmaniose visceral sugere possível participação desta espécie na cadeia de transmissão dessa parasitose em Mato Grosso.
\end{abstract}

Palavras-chaves: Leishmaniose visceral. Lutzomyia cruzi. Mato Grosso.

\begin{abstract}
An epidemic of visceral leishmaniasis began in 1998, in the Metropolitan Region of Cuiabá, the capital of the State of Mato Grosso, Brazil. Today, it has reached 34 (24.1\%) of the 141 municipalities in the state. Between January 1998 and December 2005, 138 autochthonous cases were notified, mainly in males (58\%), children aged 0-9 years (51.5\%) and inhabitants of urban areas (66.7\%). Canine visceral leishmaniasis has been detected in 41 municipalities, with positive serum in 9\% of the 40,000 dogs examined. Lutzomyia longipalpis and/or Lutzomyia cruzi were captured in 14 out of the 18 municipalities that simultaneously recorded both human and canine visceral leishmaniasis. These findings indicate that visceral leishmaniasis transmission bas become disseminated throughout the state, following migratory flows and the process of disorderly occupation of urban areas. The presence of Lutzomyia cruzi alone in areas with high incidence of human and canine cases suggests possible participation by this species in the transmission chain for visceral leishmaniasis in Mato Grosso.
\end{abstract}

Key-words: Visceral leishmaniasis. Lutzomyia cruzi. Mato Grosso.

Os primeiros registros de casos autóctones humanos de leishmaniose visceral (LV) em Mato Grosso ocorreram em 1973, no município de Guiratinga, Sudeste do estado, tendo sido diagnosticados oito casos, sugerindo que a infecção se restringia ao ambiente silvestre de transmissão $0^{3}$. Durante as duas décadas seguintes, a transmissão da LV em Mato Grosso foi considerada esporádica e sua autoctonia muitas vezes questionada. No período de 1992 a 1994, quatro novos casos da doença foram diagnosticados na região Centro-Sul ${ }^{11}$, e somente em 1998 registrou-se o primeiro caso de IV na Região Metropolitana de Cuiabá. Esta transmissão urbana teve início no município de
Várzea Grande, vizinho à capital ${ }^{6}$, estendendo-se em poucos anos para municípios de distintas regiões geográficas do estado, incluindo Cuiabá.

Semelhante à infecção humana, são recentes os relatos de LV canina e da presença de Lutzomyia longipalpis em Mato Grosso. Cães sorologicamente positivos foram detectados em 1997 e 1998, em áreas urbanas dos municípios de Cuiabá ${ }^{21}$ e Várzea Grande ${ }^{16}$, respectivamente. Os levantamentos entomológicos realizados àquela época demonstraram a presença de $L$. longipalpis nas áreas de transmissão humana e canina da doença ${ }^{27}$.

\footnotetext{
1. Pós-graduação em Ciências da Saúde, Faculdade de Ciências Médicas da Universidade Federal de Mato Grosso. Cuiabá, MT. 2. Núcleo de Estudo de Doenças Infecciosas e

Tropicais de Mato Grosso, Faculdade de Ciências Médicas da Universidade Federal de Mato Grosso. Cuiabá, MT.

Endereço para correspondência: Dr. Gustavo Mestre. R. Cel. Evaristo da Costa e Silva 66, CPA 1, 78055-181 Cuiabá, MT.

Tel: 5565 3641-2601; Fax: 5565 3617-1681.

e-mail: gmestre@terra.com.br

Recebido para publicação em 4/4/2006

Aceito em 12/1/2007
} 
Entendendo a atual importância da LV em Mato Grosso, é inquestionável a necessidade de grande esforço para o conhecimento de sua dinâmica de transmissão, bem como dos grupos populacionais de maior risco. Assim sendo, delineou-se o presente estudo para descrever a expansão da epidemia da LV no estado, explicitar a sua atual magnitude epidemiológica e apresentar as características de sua distribuição, com vistas a orientar o serviço de saúde pública para uma melhor vigilância e controle dessa parasitose.

\section{MATERIAL E MÉTODOS}

Área de estudo. O Estado de Mato Grosso possui 141 municípios e população de 2.803.272 habitantes, sendo $79,4 \%$ residentes em áreas urbanas. A capital Cuiabá está situada na região Centro-Sul e possui uma população de 533.800 habitantes. O município vizinho de Várzea Grande, com população de 248.728 habitantes compõe, com a capital, a chamada Região Metropolitana de Cuiabá. Próximos a essa Região se encontram vários municípios de pequeno porte, todos eles muito influenciados pelas atividades urbanas da capital e, por possuírem características históricas, geográficas e culturais semelhantes, são considerados, em conjunto, como pertencentes à Baixada Cuiabana (Figura 1).
Fontes dos dados. O número absoluto e os dados demográficos e epidemiológicos dos casos humanos de LV ocorridos em Mato Grosso no período de janeiro de 1998 a dezembro de 2005 foram obtidos no SINAN, MT ${ }^{30}$. Dados referentes aos municípios e sua população foram consultados nos arquivos do $\mathrm{IBGE}^{12}$. A Coordenadoria de Vigilância Ambiental da Secretaria de Estado de Saúde de Mato Grosso (CVA/SES-MT) contribuiu com dados relativos aos inquéritos sorológicos caninos e entomológicos, realizados entre 1998 e 2005 , prioritariamente nos municípios com casos humanos registrados. O Laboratório Central de Saúde Pública do Estado de Mato Grosso (MT - Laboratório) realizou o diagnóstico da $\mathrm{LV}$ canina através da triagem pelo método imunoenzimático (ELISA) e confirmação pela reação de imunofluorescência indireta (RIFI), conforme preconizado pelo Ministério da Saúde ${ }^{17}$, sendo considerado positivo o soro que apresentou reação em diluição igual ou superior ao ponto de corte de 1:40. Para a realização das capturas dos flebotomíneos foram utilizadas armadilhas luminosas do tipo CDC, instaladas no entardecer e recolhidas cerca de 12 horas depois, ao amanhecer. As armadilhas foram colocadas, preferencialmente, nos bairros de residência dos casos humanos ou com maior prevalência de infecção canina. Os insetos foram capturados tanto dentro das residências (intradomiciliar) quanto nos anexos e/ou abrigos de animais domésticos (peridomiciliar).

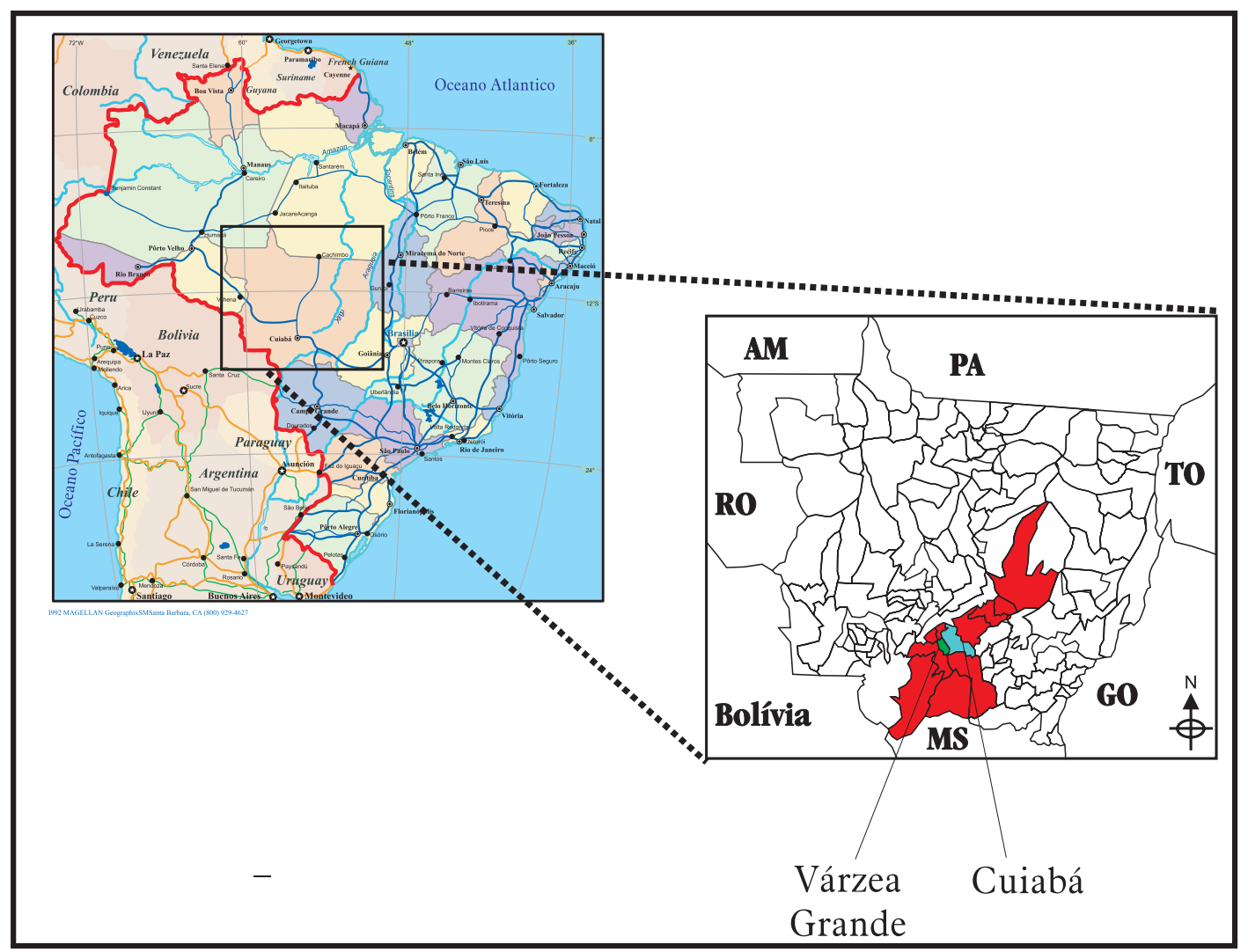

Figura 1 - Mapa do Brasil, destacando o Estado de Mato Grosso, a Região Metropolitana de Cuiabá,formada pelos municípios de Cuiabá e Várzea Grande, e os municípios da Baixada Cuiabana. 
Após triagem, os espécimes foram clarificados, montados e identificados ${ }^{33}$. Exemplares estão depositados no Laboratório de Entomologia da Secretaria Estadual de Saúde de Mato Grosso. Os flebotomíneos capturados foram encaminhados ao Laboratório de Entomologia da Fundação Nacional de Saúde (1998-2001) e ao Núcleo de Entomologia da CVA/SES-MT, para identificação (2002-2005).

Considerações éticas. 0 estudo foi aprovado pelo Comitê de Ética e Pesquisa do Hospital Universitário Júlio Müller da Universidade Federal de Mato Grosso, conforme Parecer No 343/CEP-HUJM/05.

\section{RESULTADOS}

0 ressurgimento da leishmaniose visceral em Mato Grosso. A epidemia de LV em Mato Grosso teve início em janeiro de 1998, com o registro de 13 casos humanos, todos autóctones e procedentes do município de Várzea Grande. Os bairros São Mateus e Jardim Eldorado, situados em uma mesma faixa periurbana, foram os mais atingidos e representaram, no início da epidemia, áreas de degradação ambiental provocadas pela ocupação desordenada de famílias de baixa renda, popularmente conhecidas como áreas de invasão ou grilo. Para uma região sabidamente indene, esses 13 casos caracterizaram franca epidemia da doença no município, que culminou com uma incidência de 6,2/100.000 habitantes naquele ano. A transmissão persistiu e, no ano de 1999, a incidência da LV naquele município chegou a 11,2/100.000 habitantes, quase duas vezes maior do que a de 1998 .

A partir dos primeiros casos notificados em Várzea Grande, a transmissão de $\mathrm{LV}$ se expandiu para outros municípios de Mato Grosso. Durante o período de janeiro de 1998 e dezembro de 2005, 138 novos casos confirmados da doença foram notificados em todo o estado, resultando em coeficientes de incidência acumulada e letalidade de 5,4 casos/100.000 habitantes e 11,6\%, respectivamente (Tabela 1). Predominou o sexo masculino (58\%) e a faixa etária de $0-9$ anos (51,5\%). Em geral, o tempo de início de sintomatologia referido pelos pacientes foi alto, variando de 7 a 300 dias e com média (DP) de 56,8 $(51,1)$ dias. Todos os pacientes tiveram o diagnóstico confirmado com o auxílio de recursos laboratoriais, sendo a pesquisa parasitológica realizada em $98 \%$ dos casos. A coinfecção Leishmania-HIV foi observada em três pacientes.

Do total notificado, 118 (85,5\%) foram confirmados como autóctones do município de residência. Contudo, todos os 138 pacientes foram infectados dentro do Estado de Mato Grosso, com distribuição mensal irregular no período avaliado, não se caracterizando padrão sazonal de transmissão. No decorrer de 2001 a 2003 observou-se redução progressiva na identificação de casos novos. Entretanto, a partir de 2004, importante aumento do número de casos foi observado. A transmissão da LV foi mais elevada na área urbana (66,7\%) do que na rural $(33,3 \%)$ em quase todos os anos do período do estudo (Figura 2).
Tabela 1 - Incidência acumulada e letalidade anuais de leishmaniose visceral humana em Mato Grosso, 1998-2005.

\begin{tabular}{lccc}
\hline Ano & $\begin{array}{c}\text { Coeficiente de incidência } \\
\text { (por 100.000 habitantes) }\end{array}$ & $\begin{array}{c}\text { Óbitos } \\
\left(\mathrm{n}^{\mathrm{o}}\right)\end{array}$ & $\begin{array}{c}\text { Coeficiente de letalidade } \\
(\%)\end{array}$ \\
\hline 1998 & 0,56 & 2 & 15,4 \\
1999 & 1,09 & 2 & 7,7 \\
2000 & 0,92 & 3 & 13,0 \\
2001 & 0,62 & - & - \\
2002 & 0,38 & 3 & 30,0 \\
2003 & 0,41 & - & - \\
2004 & 0,70 & 3 & 15,8 \\
2005 & 0,71 & 3 & 15,0 \\
\hline Total & $\mathbf{5 , 4 0}$ & $\mathbf{1 6}$ & $\mathbf{1 1 , 6}$ \\
\hline
\end{tabular}

Fonte: SINAN-MT

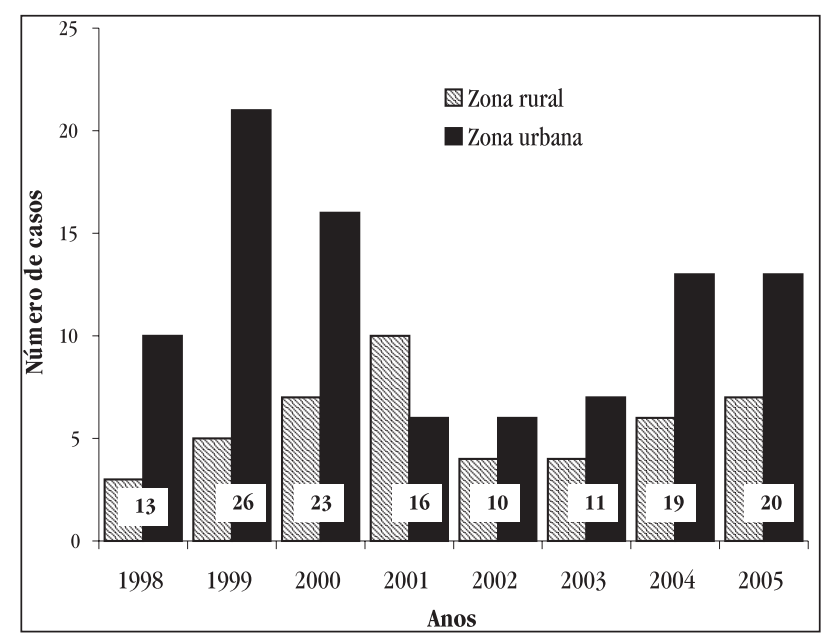

(Fonte: SINAN, MT)

Figura 2 - Diagrama comparativo entre o número de casos de pacientes positivos para leishmaniose visceral, procedentes de zona rural ou urbana, notificados em Mato Grosso, 1998-2005.

A expansão da leishmaniose visceral humana em Mato Grosso. No biênio de 1998 a 1999, 39 casos de LV foram notificados no estado, restringindo-se a apenas três municípios da Baixada Cuiabana (Várzea Grande, Chapada dos Guimarães e Nossa Senhora do Livramento). Nos anos subseqüentes, a LV passou a ser registrada em vários outros municípios e, em 2005, a transmissão humana encontrava-se distribuída em 34 (24,1\%) dos 141 municípios matogrossenses, com maior concentração de casos nas regiões Centro-Sul e Sudeste do estado. A magnitude da expansão geográfica da transmissão de LV em Mato Grosso pode ser observada na Figura 3 e Tabela 1.

0 município de Várzea Grande foi o que mais notificou a doença, com $50(36,2 \%)$ casos no período, dos quais 37 (74\%) ocorreram nos dois primeiros anos da epidemia. A primeira ocorrência de LV na capital, Cuiabá, só aconteceu em 2005, com registro de cinco $(3,6 \%)$ casos durante 0 ano. A infecção humana também foi elevada nos municípios de Poxoréu com $16(11,6 \%)$ casos, Barra do Garças com 14 (10,1\%) casos e Jaciara com 8 (5,8\%) casos, localizados na Região Sudeste do Estado. Nos demais municípios a incidência foi baixa, não ultrapassando quatro casos durante os oito anos avaliados. 


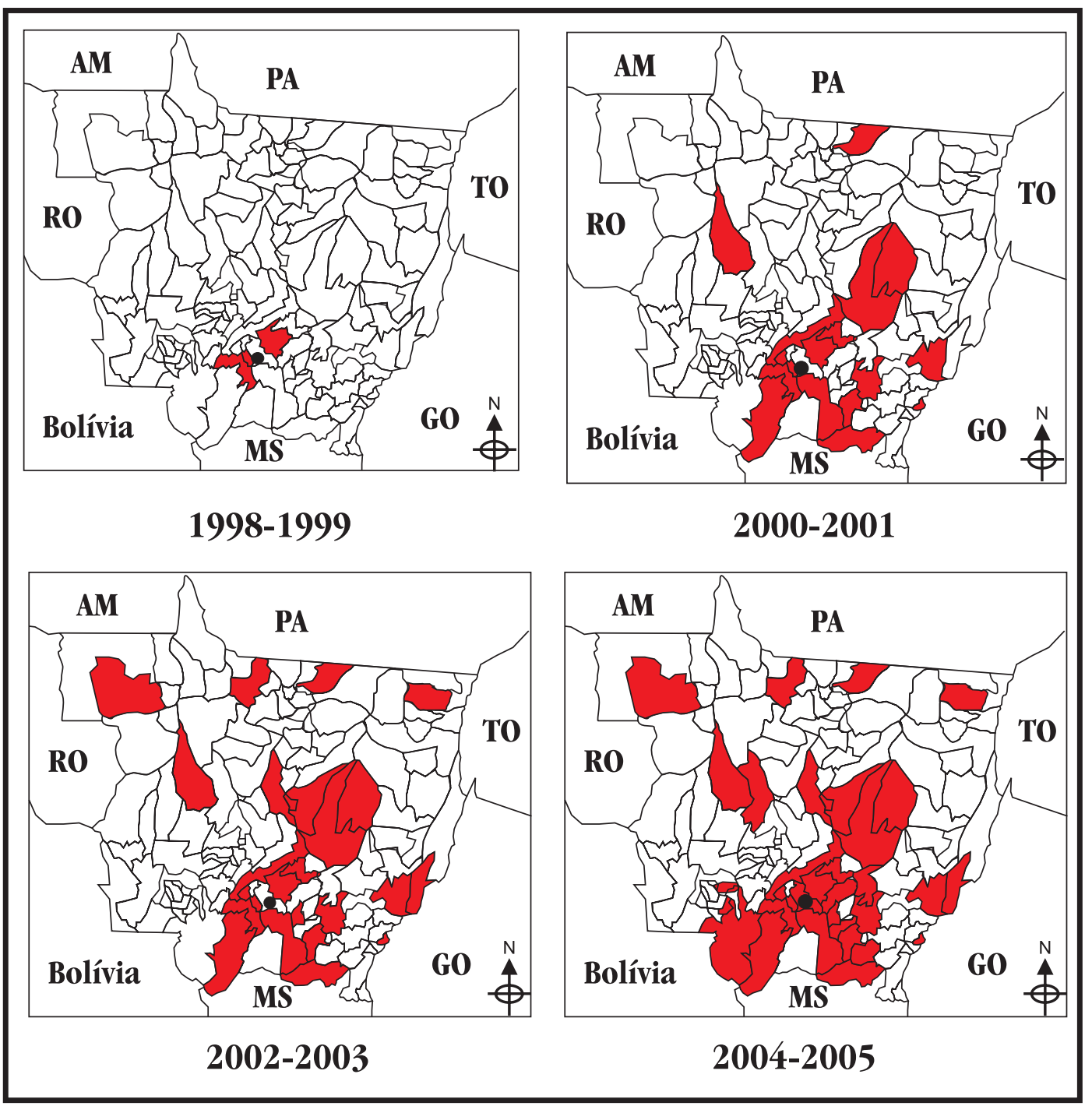

Fonte: SINAN, MT)

Figura 3 - Expansão territorial da leishmaniose visceral humana no Estado de Mato Grosso, segundo biênios de ocorrência, 1998-2005 (casos acumulados).

Evidência da dispersão da leishmaniose visceral canina em Mato Grosso. De acordo com os dados da CVA/SESMT, no período de 1998 a 2005, foram realizados inquéritos caninos em áreas urbanas e peri-urbanas de 49 municípios matogrossenses. Em 41 (83,7\%) deles, foram encontrados cães sorologicamente positivos para leishmanioses, dos quais 18 concentraram $87,7 \%$ dos casos da LV humana notificados no estado (Figura 4A). Foram avaliados 40.000 cães em todo 0 estado, resultando em 9\% de soropositividade para leishmaniose canina, no período de 1998 a 2005. 0 município de Jaciara apresentou o maior registro de cães positivos, revelando uma taxa de infecção de $40 \%$.

A presença de vetores da leishmaniose visceral em ampla extensão geográfica de Mato Grosso. Os levantamentos entomológicos foram realizados no período de 1998 a $2005 \mathrm{em}$ 68 municípios matogrossenses, entre os quais se incluíram os 34 municípios com casos humanos de LV. Em 32 municípios foram identificadas espécies vetoras da Leishmania chagasi. Presença isolada de L. longipalpis e de L. cruzi foi registrada em 9 e 8 municípios, respectivamente. Esses vetores foram identificados em 14 dos 18 municípios com transmissão concomitante da LV humana e canina (Figura 4B). Chama a atenção a ocorrência de $L$. cruzi em 11 (78,6\%) dos 14 municípios com registro simultâneo de LV humana e canina (Tabela 2).

Tabela 2 - Distribuição dos municípios com superposição de registros de casos humanos e caninos de leishmaniose visceral e as espécies de flebotomíneos encontradas, Mato Grosso, 1998-2005.

\begin{tabular}{lccc}
\hline & \multicolumn{3}{c}{$\begin{array}{c}\text { Municípios com registro } \\
\left(\mathrm{n}^{0}\right)\end{array}$} \\
\cline { 2 - 4 } & & & LV humana + \\
Espécies vetoras encontradas & LV humana & LV canina & LV canina \\
\hline Lutzomyia longipalpis & 2 & 2 & 3 \\
Lutzomyia cruzi & 1 & 0 & 4 \\
Lutzomyia longipalpis + L. cruzi & 2 & 2 & 7 \\
\hline
\end{tabular}

LV: leishmaniose visceral

Fonte: SINAN-MT 


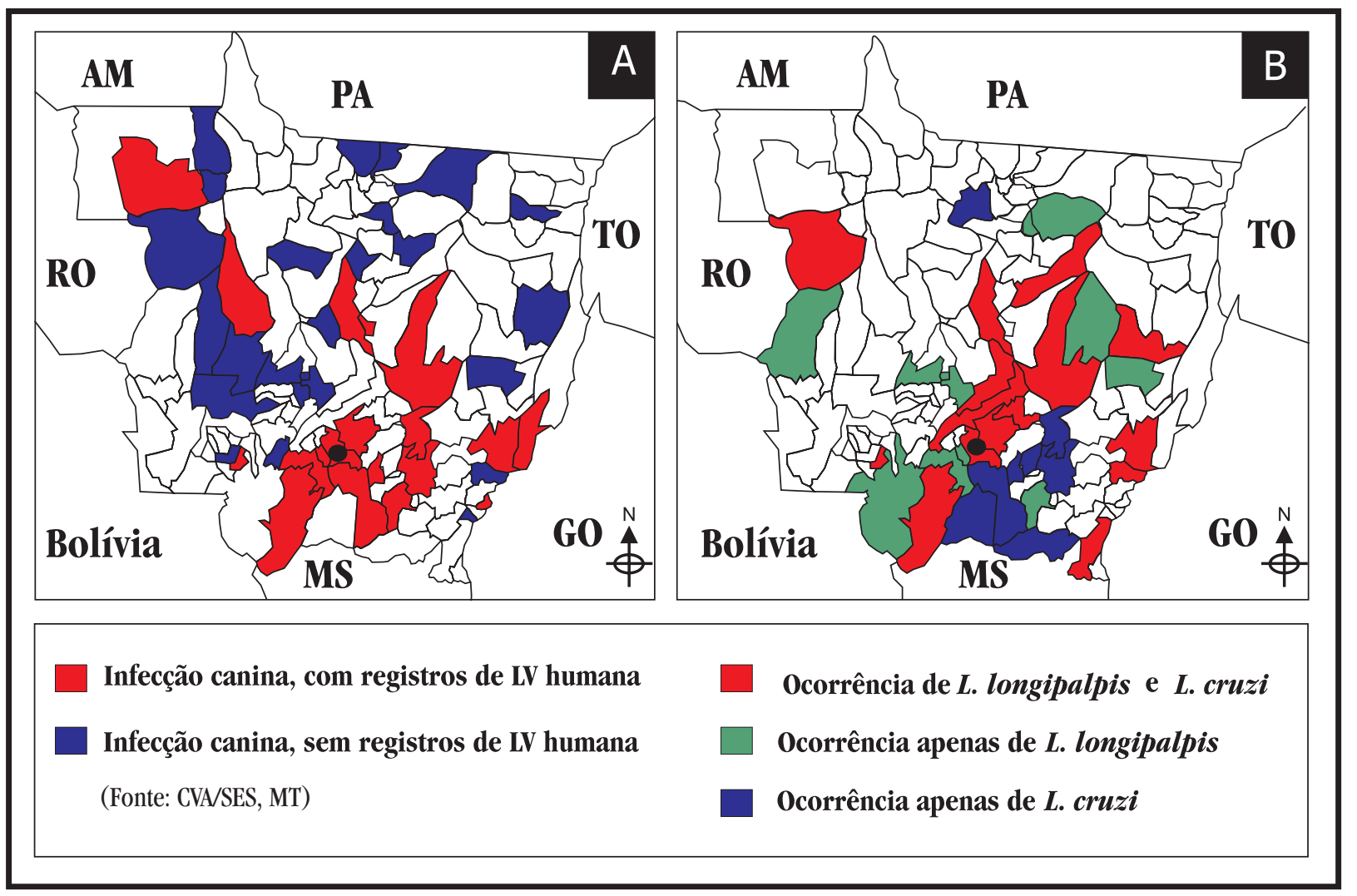

Figura 4 - Mapas do Estado de Mato Grosso mostrando municípios com ocorrência de infecção canina (A) e de espécies de flebotomíneos vetoras da leishmaniose visceral (B), 1998-2005.

\section{DISCUSSÃo}

Os resultados do presente estudo revelaram que, no período compreendido entre janeiro de 1998 e dezembro de 2005, 138 novos casos de LV humana foram registrados em Mato Grosso. A alta magnitude inicial da epidemia fez com que Várzea Grande, município da Região Metropolitana de Cuiabá fosse classificado em 2003, pelo Departamento de Vigilância Epidemiológica do Ministério da Saúde, como área de transmissão intensa de LV no Brasil, juntamente com os municípios de Campo Grande e Corumbá, MS, Palmas, TO, Araçatuba, SP, Belo Horizonte e Montes Claros, MG, São Luis, MA e Teresina, PI ${ }^{18}$.

A transmissão da LV em Mato Grosso progrediu em franca expansão geográfica, atingindo outros municípios do Centro-Sul do estado, região caracterizada por apresentar grande mobilidade populacional. Posteriormente, novos focos de transmissão foram detectados em municípios contíguos à Baixada Cuiabana, seguindo pelos corredores de circulação do estado, especialmente em direção ao Sudeste e Norte mato-grossenses. Padrão semelhante de transmissão foi observado no interior de São Paulo, onde a LV foi detectada em zona urbana de vários municípios, como Araçatuba, Bauru, Marília e Presidente Prudente, acompanhando os eixos rodoviários e ferroviários do estado ${ }^{4}$. A expansão da LV em Mato Grosso pode ter sido facilitada pela grande rede de estradas vicinais presentes nas regiões Centro-Sul e Sudeste do estado, contribuindo para o estabelecimento de correntes migratórias entre seus municípios e a Baixada Cuiabana. Da mesma forma, os grandes investimentos do acesso rodoviário entre a capital e os municípios do Norte mato-grossense ocorridos nas últimas décadas, a exemplo da recuperação e ampliação da BR-163, intensificaram o deslocamento de pessoas e mercadorias no sentido sul-norte do estado ${ }^{2}$.

0 aumento da densidade do vetor, o convívio muito próximo do homem com o reservatório doméstico, o desmatamento acentuado e a constante mobilização de pessoas constituem os principais determinantes dos níveis epidêmicos da LV nos grandes centros urbanos ${ }^{13}$. Na década de 1980, o município de São Luis do Maranhão recebeu grande fluxo migratório provocado pelo deslocamento de lavradores que fugiam da seca no Piauí e no Ceará, levando ao registro de 1.089 casos de LV naquela capital em 15 anos ${ }^{15}$. 0 êxodo rural e a intensa migração ocorridos nas últimas duas décadas em Mato Grosso também promoveram o crescimento desordenado de cidades como Cuiabá, Várzea Grande, Barra do Garças e Rondonópolis ${ }^{26}$ e, certamente, contribuíram para o surgimento da epidemia atual de LV. Adicionalmente, a introdução de cães infectados em determinadas áreas pode estar associada à migração, uma vez que estes animais, muitas vezes, são trazidos infectados da região de origem, contribuindo para a infecção dos flebotomíneos presentes em áreas de desmatamento recente e em zonas peri-urbanas ${ }^{13}$.

Sherlock ${ }^{31}$ observou na Bahia e em outras regiões do país que a pobreza, a desnutrição e a alta densidade de flebotomíneos, tanto no intradomicílio como no peridomicílio, estão associadas à elevada presença de animais domésticos, péssimas condições sanitárias e baixo nível sócio-econômico em áreas de transmissão 
da leishmaniose visceral. Esse quadro é bem ilustrado pelo município matogrossense de Várzea Grande, cujo surgimento de núcleos residenciais nas periferias urbanas do município, decorrentes da imigração de população de baixa renda, proporcionaram condições favoráveis para a transmissão da LV, como a destruição de ecótopos silvestres, o intenso convívio do homem com animais domésticos e as precárias condições de moradia, de coleta de lixo e de saneamento básico.

A epidemia de LV em Mato Grosso vem acometendo principalmente o sexo masculino e crianças na faixa etária inferior a cinco anos, similar ao observado no Estado de Rio de Janeiro $^{14}$, Alagoas ${ }^{25}$ e na Região Metropolitana de Belo Horizonte ${ }^{32}$. A concentração de casos nessa faixa etária reforça a observação de que a transmissão da LV é mais comum nos ambientes peridomiciliar e intradomiciliar ${ }^{2232}$.

A distribuição mensal dos casos de $\mathrm{LV}$ não foi homogênea durante o período estudado. Outros autores também não verificaram correlação entre as médias climáticas mensais de temperatura e precipitação com o registro de casos de IV no Brasil ${ }^{1014}$. Isso deve ocorrer devido ao tempo de incubação da doença, que é variável de doente para doente, assim como 0 tempo que o enfermo leva para buscar assistência médica após ter sido infectado.

A média de 56 dias para o diagnóstico da LV e a alta letalidade observada em Mato Grosso pode ser consequiência do despreparo dos serviços de saúde para o diagnóstico e tratamento da doença. Taxas semelhantes de letalidade foram descritas no Estado de São Paulo ${ }^{4}$ e na Região Metropolitana de Belo Horizonte ${ }^{8}$ e sugere que a falta de informação da população e principalmente dos profissionais de saúde sobre a LV têm, provavelmente, retardado o diagnóstico dos casos, contribuindo para a sua maior gravidade.

A leishmaniose visceral canina foi identificada em ampla extensão geográfica de Mato Grosso, atingindo áreas urbanas e peri-urbanas de vários municípios, resultando em soropositividade média de $\%$ no período estudado. A frequiência observada está de acordo com a encontrada em várias localidades do país, oscilando entre $1,9 \%$ e $35 \%$ em diferentes estudos ${ }^{7}{ }^{23}$ e que geralmente ocorre em concomitância com o surgimento de epidemias urbanas, como as descritas nos municípios de Araçatuba (SP) e Montes Claros (MG), cujos inquéritos sorológicos caninos revelaram prevalências de $12,1 \%$ e $5 \%$, respectivamente ${ }^{520}$.

A ocorrência de L. longipalpis tem sido amplamente associada ao aparecimento de casos humanos ou caninos de $\mathrm{LV}^{28}$. A presença do vetor da Leishmania chagasi $i$ a periferia de cidades que registraram grande número de casos de LV humana e elevadas taxas de infecção canina confirmam a transmissão urbana da endemia em Mato Grosso, tal como observado em vários outros municípios brasileiros, onde foram identificados vetores para LV coincidindo com o crescente número de casos humanos e caninos da doença ${ }^{4242832}$. Ressalta-se, entretanto, a presença isolada e alta frequiência de ocorrência de $L$. cruzi em municípios matogrossenses que notificaram casos humanos e caninos simultâneos de LV, sugerindo possível incriminação desta espécie na transmissão desta parasitose nessas áreas, como já anteriormente sugerida para Mato Grosso ${ }^{19}$ e Mato Grosso do $\mathrm{Su}^{29}$.

Os resultados deste estudo indicam que a transmissão da LV em Mato Grosso continua em expansão para o interior do estado e que sua disseminação acompanha o processo de ocupação urbana desordenada, aliado ao fluxo migratório entre os municípios da região Centro-Sul com o Norte e Sudeste matogrossenses. A alta frequiência de $L$. cruzi em municípios com alta incidência de casos humanos e caninos de LV sugere possível participação desta espécie na cadeia de transmissão da parasitose em nosso meio e merece ser avaliada em estudos entomológicos projetados especialmente para confirmar esta hipótese.

\section{AGRADECIMENTOS}

Aos profissionais das Coordenadorias de Vigilância Epidemiológica e de Vigilância em Saúde Ambiental da Secretaria de Estado da Saúde de Mato Grosso, pela disponibilidade dos dados epidemiológicos, caninos e entomológicos de leishmaniose visceral.

\section{REFERÊNCIAS}

1. Alexander B, Carvalho RL, McCallum H, Pereira MH. Role of the domestic chicken (Gallus gallus) in the epidemiology of urban visceral leishmaniasis in Brazil. Emerging Infectious Diseases 8: 1480-1485, 2002.

2. Alves Júnior GT. 0 planejamento governamental e seus reflexos na estrutura fundiária de Mato Grosso. Caminhos de Geografia 4: 17-30, 2003.

3. Baruffa G, Cury P. Contribuição ao estudo do calazar em Mato Grosso. Revista de Patologia Tropical 2: 345-361, 1973.

4. Camargo-Neves VLF. Leishmaniose visceral americana: Doença emergente no estado de São Paulo. Comciência - Revista Eletrônica de Jornalismo Científico. Disponível em < http://www.comciencia.br/ reportagens/2005/06/17.shtml>, 2005.

5. Camargo-Neves VLF, Katz G, Rodas LAC, Poletto DW, Lage LC, Spínola RMF, Cruz OG. Utilização de ferramentas de análise espacial na vigilância epidemiológica de leishmaniose visceral americana - Araçatuba, São Paulo, Brasil, 1998-1999. Cadernos de Saúde Pública 17: 1263-1267, 2001.

6. Camiá RP, Rinaldi J, Fontes CJF, Hueb, H. Foco de leishmaniose visceral em Mato Grosso. Revista da Sociedade Brasileira de Medicina Tropical 32 (supl II): 127$128,1999$.

7. Evans TG, Vasconcelos IAB, Lima JW, Teixeira JM, Macrullife IT, Lopes UG, Pearson $\mathrm{RD}$, Vasconcelos AW. Canine visceral leishmaniasis in northeast Brazil: Assessment of serodiagnosis methods. American Journal of Tropical Medicine and Hygiene 42: 118-123, 1990.

8. Filogonio LSB, Rabello A, Luz ZMP. Fatores associados à evolução clínica e letalidade da leishmaniose visceral em pacientes atendidos em Belo Horizonte. In: Resumos da XXI Reunião de Pesquisa Aplicada em doença de Chagas e Leishmanioses, Uberaba, MG, 2005.

9. França-Silva JC, Costa RT, Siqueira AM, Machado-Coelho GLL, Costa CA, Mayrink W, Vieira EP, Costa JS, Genaro 0, Nascimento E. Epidemiology of canine visceral leishmaniasis in the endemic area of Montes Claros municipality, Minas Gerais state, Brazil. Veterinary Parasitology 111: 161-173, 2003.

10. Gurgel HC, Bavia ME, Carneiro DDMT, Silva CEP, Madureira Filho C, Rios RB, Barbosa MG. A contribuição do NDVI para o estudo epidemiológico da Leishmaniose Visceral Americana, no interior da Bahia. In: Anais do XII Simpósio Brasileiro de Sensoriamento Remoto, Goiânia, GO p. 2673-2680, 2005.

11. Hueb M, Assis SB, Guimarães EED, Rosa DL, Fontes CJF. Ocorrência de transmissão autóctone de leishmaniose visceral em Mato Grosso. Revista da Sociedade Brasileira de Medicina Tropical 29: 281-282, 1996. 
12. Instituto Brasileiro de Geografia e Estatística. Estimativa da população em 01.07.2005. Disponível em <http://www.ibge.gov.br>, 2005.

13. Marzochi MCA, Marzochi KBF. Leishmanioses em áreas urbanas. Revista da Sociedade Brasileira de Medicina Tropical 30: 162-164, 1997.

14. Marzochi MCA, Marzochi KBF, Carvalho RW. Visceral leishmaniasis in Rio de Janeiro. Parasitology Today 10: 37-40, 1994.

15. Mendes WS, Silva AAM, Trovão JR, Silva AR, Costa JML. Expansão espacial da leishmaniose visceral americana em São Luis, Maranhão, Brasil. Revista da Sociedade Brasileira de Medicina Tropical 35: 227-231, 2002.

16. Mestre GLC, Fontes CJF. Perfil epidemiológico da leishmaniose visceral (calazar) na área urbana de Várzea Grande, Mato Grosso, 1998-2000. Revista Saúde e Ambiente 5: 97, 2002.

17. Ministério da Saúde. Manual de Vigilância e Controle da Leishmaniose Visceral, Secretaria de Vigilância em Saúde, Departamento de Vigilância Epidemiológica, Brasília, 2004

18. Ministério da Saúde. Vigilância e controle da leishmaniose visceral. Classificação dos municípios para a vigilância e controle da leishmaniose visceral, Brasil, no período de 1998 a 2002. Disponível em: <http://www.saude.ms.gov.br/Saude/SES/ ReuniaoLeishmanioseVisceral.htm>, 2005.

19. Missawa NA, Lima GBM. Distribuição espacial de Lutzomyia longipalpis (Lutz \& Neiva, 1912) e Lutzomyia cruzi (Mangabeira, 1938) no Estado de Mato Grosso. Revista da Sociedade Brasileira de Medicina Tropical 39: 337-340, 2006.

20. Monteiro EM, França-Silva JC, Costa RT, Costa DC, Barata RA, Paula EV, MachadoCoelho GLL, Rocha MF, Fortes-Dias CL, Dias ES. Leishmaniose visceral: Estudo de flebotomíneos e infecção canina em Montes Claros, Minas Gerais. Revista da Sociedade Brasileira de Medicina Tropical 38: 147-152, 2005.

21. Moura ST, Fernandes CGN, Pandolpho VC, Silva RR. Diagnóstico de leishmaniose canina na área urbana do município de Cuiabá, estado de Mato Grosso, Brasil. Brazilian Journal of Veterinary Research and Animal Science 36: 101-102, 1999.

22. Nascimento MDSB, Costa JML, Fiori BIP, Viana GMC, Gonçalves Filho M, Alvim AC, Bastos OC, Nakatani M, Reed S, Badaró R, Silva AR, Burattini MN. Aspectos epidemiológicos determinantes na manutenção da leishmaniose visceral no estado do Maranhão - Brasil. Revista da Sociedade Brasileira de Medicina Tropical 29: 233-240, 1996.
23. Nunes MP, Jackson JM, Carvalho RW, Furtado NJ, Coutinho SG. Serological survey for canine cutaneous and visceral leishmaniasis in area for transmission in Rio de Janeiro where prophylactic measures had been adapted. Memórias do Instituto Oswaldo Cruz 86: 411-417, 1991

24. Oliveira AG, Andrade Filho JD, Falcão AL, Brazil RP. Estudo de flebotomíneos (Díptera, Psychodidae, Phlebotominae) na zona urbana da cidade de Campo Grande, Mato Grosso do Sul, Brasil, 1999-2000. Cadernos de Saúde Pública 19: 933-944, 2003

25. Pedrosa CMS, Rocha EMM. Aspectos clínicos e epidemiológicos da leishmaniose visceral em menores de 15 anos procedentes de Alagoas, Brasil. Revista da Sociedade Brasileira de Medicina Tropical 37: 300-304, 2004.

26. Piaia II. Geografia de Mato Grosso. Edunic, Cuiabá, 1999.

27. Ribeiro ALM, Missawa NA. Spatial distribution of phlebotomine species in the state of Mato Grosso, Brazil, in the period of 1996 to 2001. Entomología y Vectores 9: 33-34, 2002

28. Santos SO, Arias JR, Hoffmann MP, Furlan MBG, Ferreira WF, Pereira C, Ferreira L. The presence of Lutzomyia longipalpis in a focus of american visceral leishmaniasis where the only proven vector is Lutzomyia cruzi. Corumbá, Mato Grosso do Sul State. Revista da Sociedade Brasileira de Medicina Tropical 36: 633-634, 2003.

29. Santos SO, Arias JR, Ribeiro AA, Hoffmann MP, Freitas RA, Malacco MAF. Incrimination of Lutzomyia cruzi as a vector of american visceral leishmaniasis. Medical and Veterinary Entomology 12: 315-317, 1998.

30. Secretaria de Estado de Saúde, Sistema de Informações de Agravos de Notificação (SINAN) Cuiabá, MT, 2005.

31. Sherlock IA. Ecological interactions of visceral leishmaniasis in the State of Bahia. Memórias do Instituto Oswaldo Cruz 91: 671-683, 1996.

32. Silva ES, Gontijo CMF, Pacheco RS, Fiúza VOP, Brazil RP. Visceral leishmaniasis in the metropolitan region of Belo Horizonte, state of Minas Gerais, Brazil. Memórias do Instituto Oswaldo Cruz 96: 285-291, 2001.

33. Young DG, Duncan MA. Guide to the identification and geographic distribuition of Lutzomyia sand flies in México, the West Indies, Central and South America (Diptera: Psychodidae). Associated Publishers American Entomological Institute. Florida, 1994. 